\title{
c-Jun transactivates Puma gene expression to promote osteoarthritis
}

\author{
HUADING LU*, GANG HOU*, YONGKAI ZHANG, YUHU DAI and HUIQING ZHAO \\ Department of Orthopaedics, The Third Affiliated Hospital, Sun Yat-sen University, Guangzhou, Guangdong, P.R. China
}

Received September 6, 2013; Accepted February 12, 2014

DOI: $10.3892 / \mathrm{mmr} .2014 .1981$

\begin{abstract}
Osteoarthritis (OA) is a chronic degenerative joint disorder in which genetic, hormonal, mechanical and ageing factors affect its progression. Current studies are focusing on chondrocytes as a key mediator of OA at a cellular level. however, the mechanism underlying chondrocyte apoptosis remains unclear. PUMA is a pro-apoptotic member of the $\mathrm{BH} 3$-only subgroup of the Bcl-2 family and is involved in a large number of physiological and pathological processes. In the present study, we examined whether PUMA has a role in IL-1 $\beta$-induced apoptosis and whether the c-Jun $\mathrm{N}$-terminal kinase (JNK)/c-Jun pathway mediates the induction of PUMA, thus contributing to chondrocyte apoptosis. The results demonstrated an increase in PUMA protein and mRNA levels in cultured mouse chondrocytes following $4 \mathrm{~h}$ of IL-1 $\beta$ treatment. Furthermore, this upregulation of PUMA was critical for chondrocyte apoptosis as knockdown of PUMA using PUMA-specific siRNA significantly reduced apoptosis in cultured cells. Upon pharmacological inhibition of the JNK/c-Jun pathway with CE11004 or SP600125, the expression of PUMA was notably suppressed with a concomitant decrease in apoptosis observed in IL- $1 \beta$-treated chondrocytes. Also, immunohistochemical studies revealed that the PUMA and c-Jun proteins were upregulated in chondrocytes from the articular cartilage of OA patients. Together, these data suggest a role for PUMA and the JNK/c-Jun pathway in the regulation of chondrocyte apoptosis during OA.
\end{abstract}

\section{Introduction}

Osteoarthritis (OA) is the most widespread degenerative joint disease affecting articular cartilage and subchondral bone. Individuals suffering from OA often experience chronic pain, tenderness, restriction of movement, crepitus and limited

Correspondence to: Dr Huading Lu, Department of Orthopedics, The Third Affiliated Hospital of Sun Yat-sen University, No. 600 Tianhe Road, Guangzhou, Guangdong 510630, P.R. China E-mail: luhuadingsci@163.com

*Contributed equally

Key words: JNK/c-Jun, PUMA, osteoarthritis, apoptosis intraarticular inflammation (1). The etiology of OA is a complex process centered on the disruption of anabolic-catabolic pathways in the bodily tissues, induced by a number of biochemical, biomechanical, and genetic factors (2). One key event in the development of OA that has garnered much attention, is the induction of apoptosis within chondrocytes. A previous study by Hashimoto et al linked chondrocyte apoptosis in human OA to cartilage degradation following the examination of the apoptotic rates via in situ and in vitro methods (3). Furthermore, Heraud et al observed that $18-21 \%$ of chondrocytes from $\mathrm{OA}$ cartilage exhibited apoptotic features compared with $2-5 \%$ in normal cartilage (4). Based on this evidence and other studies, investigations are now focusing on the chondrocyte, as well as the expression of cytokines, cell signaling molecules and pro-apoptotic proteins, as mediators of OA pathogenesis.

Interleukin-1 $\beta$ (IL-1 $\beta$ ) is a pro-inflammatory cytokine secreted by chondrocytes that has been linked to cartilage degradation in OA (5). In 2000, Heraud et al demonstrated that IL-1 $\beta$ increased the percentage of apoptotic cells in both normal and OA cartilage in a dose-dependent manner (4). IL-1 $\beta$ was also demonstrated to promote mitochondrial dysfunction and energy depletion in mouse chondrocyte-like ATDC5 cells following $48 \mathrm{~h}$ of treatment (6). Furthermore, LopezArmada et al described an upregulation of pro-apoptotic Bcl-2 family proteins in human articular chondrocytes in response to IL-1 $\beta$ (7). In the present study, due to its apoptosis-inducing effect on chondrocytes, the IL-1 $\beta$-induced model of apoptosis was selected to investigate the mechanisms of cell death in cultured cells.

PUMA, a pro-apoptotic protein belonging to the $\mathrm{Bcl}-2$ protein family, is closely associated with mitochondrial-dependent apoptosis and serves as a major effector of p53-mediated cell death. Upregulation of PUMA has been observed in a variety of apoptosis models, including oligodendroglial cell death in toxic demyelination (8), microglia-derived TNF $\alpha$ induces apoptosis in neural precursor cells (9) and crizotinib induces apoptosis in colon cancer cells (10). Knockdown or knockout of PUMA in SH-SY5Y neuroblastoma and PC-12 cells induces a significant delay in cellular apoptosis $(11,12)$, demonstrating that PUMA-mediated apoptosis is a widespread and conserved mechanism. However, until now, there have been no studies illustrating the role of PUMA in OA.

The involvement of c-Jun N-terminal kinase (JNK) in signaling transduction pathways has been well-characterized 
in mammalian cells (13), including human articular chondrocytes (14). In particular, immunostaining studies by Fan et al demonstrated the activation of the JNK signaling cascade in cultured, normal articular chondrocytes following treatment with IL-1 $\beta$ (15). c-Jun, a member of the AP-1 transcriptional complex, is preferentially phosphorylated and activated by JNK and is central in the regulation of several matrix metalloproteinases that promote the destruction of OA chondrocytes (16-18). Of note, the JNK/c-Jun pathway has also been reported to mediate the gene expression of PUMA in neuronal and tumor apoptosis $(19,20)$. Based on these results and others, the JNK/c-Jun pathway posed as an attractive target in our investigations to elucidate the mechanism of apoptosis in chondrocytes.

In the present study, we investigated whether the JNK/c-Jun pathway was involved in the induction of PUMA and thus contributes to the pathological process of OA. The results demonstrate that PUMA levels are upregulated and mediated by JNK/c-Jun pathway during the IL-1 $\beta$-induced apoptosis of chondrocytes. These data suggest a role for PUMA and the JNK/c-Jun pathway in the regulation of chondrocyte apoptosis during $\mathrm{OA}$.

\section{Materials and methods}

Isolation and culture of human articular chondrocytes. Human articular cartilage samples from 11 patients with OA (range, 61-72 years) were obtained at the time of total knee replacement. Normal cartilage was obtained from eight post-mortem donors (range, 61-69 years) with no previous history of joint pain. Informed consent was obtained in accordance with the local ethics commission and all studies were conducted under the approval of the Research Ethics Board of The Third Affiliated Hospital of Sun Yat-sen University (Guangzhou, China). Cartilage samples were harvested and fragmented into small pieces. Following the digestion of the cartilage samples with collagenase $\mathrm{D}$, the resulting cells were incubated at $37^{\circ} \mathrm{C}$ in a $5 \% \mathrm{CO}_{2}$ humidified atmosphere in DMEM containing $10 \%$ fetal bovine serum (FBS) and $1 \%$ penicillin/streptomycin.

Isolation and culture of mouse articular chondrocytes. Articular chondrocytes were isolated from the femoral heads, femoral condyles and tibial plateau of 5- to 6-day-old C57BL/6 mice. Briefly, articular cartilage tissues were minced $\left(<1 \mathrm{~mm}^{3}\right)$, and digested with 0.3 and $0.05 \%$ collagenase $\mathrm{D}$ at $37^{\circ} \mathrm{C}$ for $45 \mathrm{~min}$ and overnight, respectively. The cell suspension was filtered through a sterile $40 \mu \mathrm{M}$ nylon mesh cell strainer and the released cells were cultured in DMEM medium supplemented with $10 \% \mathrm{FBS}$ and $1 \%$ penicillin/streptomycin at $37^{\circ} \mathrm{C}$ in a humidified $5 \% \mathrm{CO}_{2}$ atmosphere. Cell viability of isolated chondrocytes was determined by trypan blue exclusion assay. Primary cells were maintained as a monolayer culture throughout this study.

Apoptosis assays. Apoptotic cells were detected by fluorescence microscopy with cell permeable Hoechst 33258 dye (Invitrogen Life Technologies, Carlsbad, CA, USA). Following $48 \mathrm{~h}$ of transfection with siRNA fragments (Shanghai Genepharma, Co., Ltd, Shanghai, China), a subconfluent monolayer of mouse chondrocytes was subjected to stress with $10 \mathrm{ng} / \mathrm{ml} \mathrm{IL-1} \beta$ for $48 \mathrm{~h}$ and stained with Hoechst 33258 dye (5 $\mu \mathrm{g} / \mathrm{ml}$ ). The nuclear morphology of cells was examined for the presence of condensed nuclei (apoptotic cells) and images were captured on an inverted fluorescence microscope (Axio Observer Z1; Carl Zeiss AD, Germany). The percentage of apoptotic cells was calculated from a minimum of 8 fields with $>100$ cells/field in an unbiased manner and the cells were scored in a blinded manner without previous knowledge of their treatments.

Immunohistochemical analysis. Immunohistochemistry for PUMA was performed using a polymer-based technology (Envision; Dako, Glostrup, Denmark). Briefly, human articular cartilage samples were dissected and post-fixed overnight in freshly prepared $4 \%$ paraformaldehyde in PBS ( $\mathrm{pH} \mathrm{7.4)}$ ) at $4^{\circ} \mathrm{C}$. Subsequently, the tissue samples were embedded in paraffin, cut into $5 \mu \mathrm{m}$ thick sections and mounted onto gelatin-coated slides. Paraffin sections were incubated for $1 \mathrm{~h}$ at $60^{\circ} \mathrm{C}$, deparaffinized in xylenes and rehydrated in a series of graded alcohols. Following three washes in phosphate-buffered saline (PBS; $\mathrm{pH}$ 7.4), endogenous peroxidase was inactivated by incubation in $3 \% \mathrm{H}_{2} \mathrm{O}_{2}$ in methanol for $10 \mathrm{~min}$. Antigen retrieval was then performed by microwaving slides in $10 \mathrm{mM}$ sodium citrate buffer (pH 6.0) for 15 min followed by cooling to room temperature. Sections were incubated with an anti-PUMA antibody (1:100) (ab33906; Abcam, Cambridge, MA, USA) at $4^{\circ} \mathrm{C}$ overnight. Following washing in PBS, a polymer reagent was applied for $30 \mathrm{~min}$ and the expression of PUMA was visualized by incubation with diaminobenzidine tetrahydrochloride (DAB). Sections were then washed with PBS, counterstained with hematoxylin for $1 \mathrm{~min}$, dehydrated for $15 \mathrm{~min}$ and mounted in neutral gum. The expression of PUMA was evaluated by IPP (version 6.0; Media Cybernetics, Silver Spring, MD, USA) following the collection of ten digital images at 1360x1024 pixel resolution at a magnification of $\mathrm{x} 400$ using a BX51WI microscope (Olympus, Tokyo, Japan). The measurement parameters included density mean, area sum and inter ocular distance (IOD). Following calibration of the optical density, the image was converted to gray scale and the values were counted.

Western blotting and antibodies. Whole cell lysates were prepared from chondrocytes lysed in ice-cold RIPA buffer (50 mM Tris, $150 \mathrm{mM} \mathrm{NaCl}, 0.1 \%$ SDS, $0.5 \%$ sodium deoxycholate, $1 \%$ Triton $\mathrm{X}-100,10 \mathrm{mM} \mathrm{NaF}, 0.1 \mathrm{mM} \mathrm{Na}_{3} \mathrm{VO}_{4}$ and $1 \mathrm{mM}$ phenylmethylsulfonyl fluoride). Lysates were cleared by centrifugation at $30,000 \mathrm{x}$ g for $10 \mathrm{~min}$ at $4^{\circ} \mathrm{C}$ and protein concentrations were determined by an Bradford assay. A $30 \mu \mathrm{g}$ aliquot of each cell lysate was subjected to SDS-polyacrylamide gel electrophoresis (PAGE) as described by 22506026. Proteins were then transferred to polyvinylidene difluoride membranes (Millipore Corporation, Billerica, MA, USA) and membranes were probed with polyclonal antibodies specific for PUMA (diluted 1:1,000; Abcam), Bax, c-Jun and GAPDH (diluted 1:1,000; Cell Signaling Technology, Inc) overnight at $4^{\circ} \mathrm{C}$. Following washing, membranes were incubated with horseradish peroxidase-conjugated secondary antibodies (dilution, 1:1,000; Jackson ImmunoResearch, West Grove, PA, USA) for $60 \mathrm{~min}$ and the proteins were visualized using the ECL chemiluminescence system (Forevergen Bioscience, China). 
A

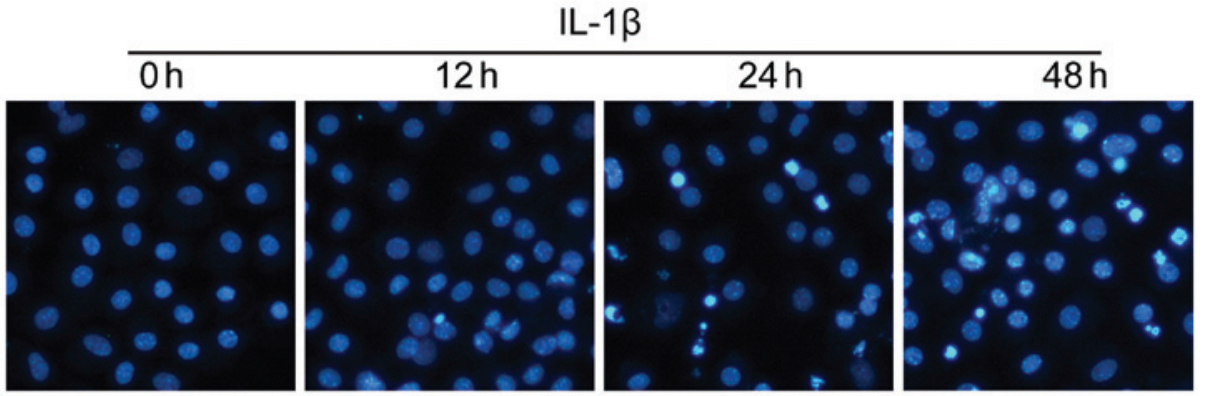

B

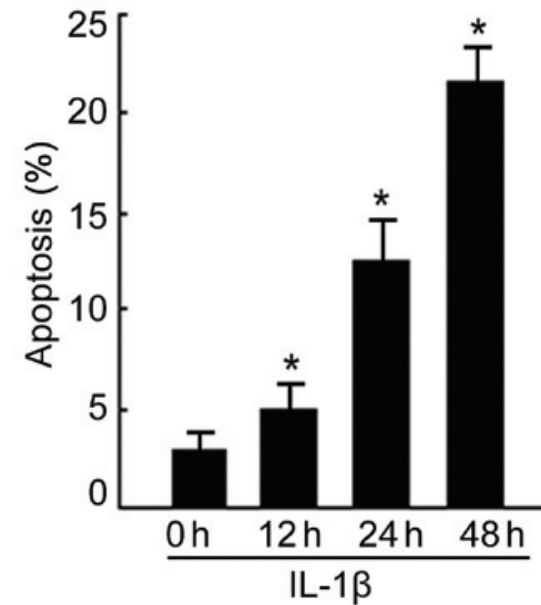

C

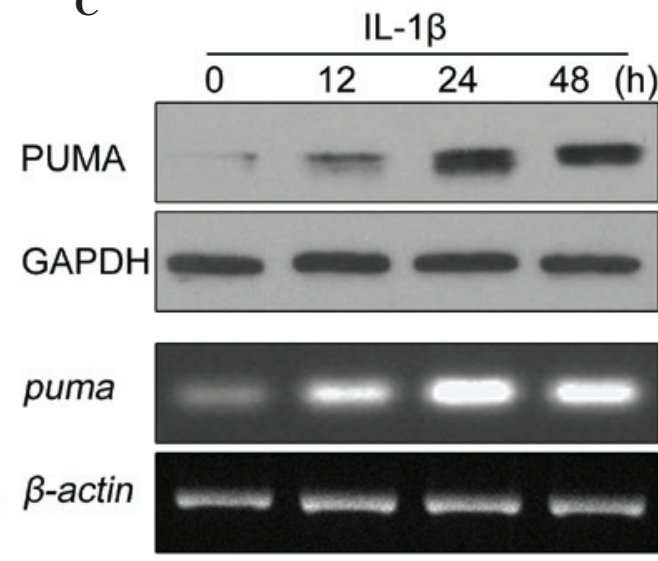

Figure 1. IL-1 $\beta$ induces upregulation of PUMA in primary cultured mouse chondrocytes. (A) Articular chondrocytes were isolated from the femoral heads, femoral condyles and tibial plateau of 5- to 6-day-old C57BL/6 mice. Subconfluent monolayers of cells were treated with $10 \mathrm{ng} / \mathrm{ml} \mathrm{IL}-1 \beta$ for the indicated times. Treated cells were then stained with Hoechst 33258 to visualize the condensed nucleus for apoptosis measurements. Representative images of cells undergoing apoptosis are presented. (B) Quantified statistical data are presented as the mean \pm SE of at least three separate experiments. (C) Chondrocytes were treated as described above. Cell lysates were subjected to western blotting with antibodies against PUMA and GAPDH (loading control). RT-PCR was performed using specific primers for Puma and $\beta$-actin. The data represent the mean \pm SE of three separate experiments. " $\mathrm{P}<0.05$. IL-1 $\beta$, interleukin- $1 \beta$; RT-PCR, reverse transcription-PCR.

Reverse transcription (RT)-PCR. Total RNA was extracted from isolated chondrocytes using TRIzol reagent (Invitrogen, USA) according to the manufacturer's recommendations. First strand cDNA was synthesized from $1 \mu \mathrm{g}$ of total RNA using M-MLV Reverse Transcriptase (Promega, Southampton, UK) and oligo(dB) primers. Reverse transcription was performed using SuperScript reverse transcriptase (Invitrogen Life Technologies) and oligo-dT primers. The following sense and anti-sense primers for Puma, $c$-jun, and $\beta$-actin were used: Puma: forward, 5'-AGCGGCGGAGACAAGAA-3' and reverse, 5'-CAAGTCCGTATCTCCATCAGTG-3'; $c$-jun: forward, 5'-CCTTCTACGACGATGCCCTC-3' and reverse, 5'-GGTTCAAGGTCATGCTCTGTTT-3'; $\beta$-actin: forward, 5'-GGCTGTATTCCCCTCCATCG-3' and reverse 5'-CCAGTTGGTAACAATGCCATGT-3'.

siRNA knockdown. Mouse chondrocytes were transfected with $100 \mathrm{nM}$ of siRNA against Puma using Lipofectamine reagent (Invitrogen Life Technologies) according to the manufacturer's instructions. siRNA sequences were as follows: si-PUMA-1, 5'-CCTGGAGGGTCATGTACAATCTCTT-3'; si-PUMA-2, 5'-GGAGGGTCATGTACAATCTCTTCAT-3. One scrambled siRNA was designed to serve as a siRNA transfection control with the following sequence;
5'-UGGUUUACAUGUCGACUAA-3'. The cells were lysed $72 \mathrm{~h}$ following siRNA transfection and the degree of knockdown was assessed by RT-PCR and western blot analysis.

\section{Results}

PUMA mRNA and protein levels are upregulated in $I L-1 \beta$ treated mouse chondrocytes. The apoptosis of chondrocytes has a key role in the development of OA, however, the mechanisms responsible for the induction of apoptosis remain unclear. In order to study the apoptotic signaling pathway, mouse chondrocytes were cultured and treated with $10 \mathrm{ng} / \mathrm{ml}$ of IL-1 $\beta$ to mimic the pathogenesis of OA. Since it has been demonstrated that PUMA regulates extracellular apoptotic pathways, we investigated the effects of IL-1 $\beta$ treatment on PUMA levels during chondrocyte apoptosis. As illustrated in Fig. 1A, chondrocytes underwent apoptosis after $48 \mathrm{~h}$ of treatment with IL-1 $\beta$. The apoptotic rate was quantified following Hoechst staining and demonstrated that to be $23 \%$ in treated chondrocytes, however only $3 \%$ in untreated cells (Fig. 1B). To determine PUMA protein and mRNA levels, IL-1 $\beta$-treated chondrocytes were then subjected to western blotting and RT-PCR. As illustrated in Fig. 1C, the PUMA protein and mRNA levels were significantly increased throughout the time 

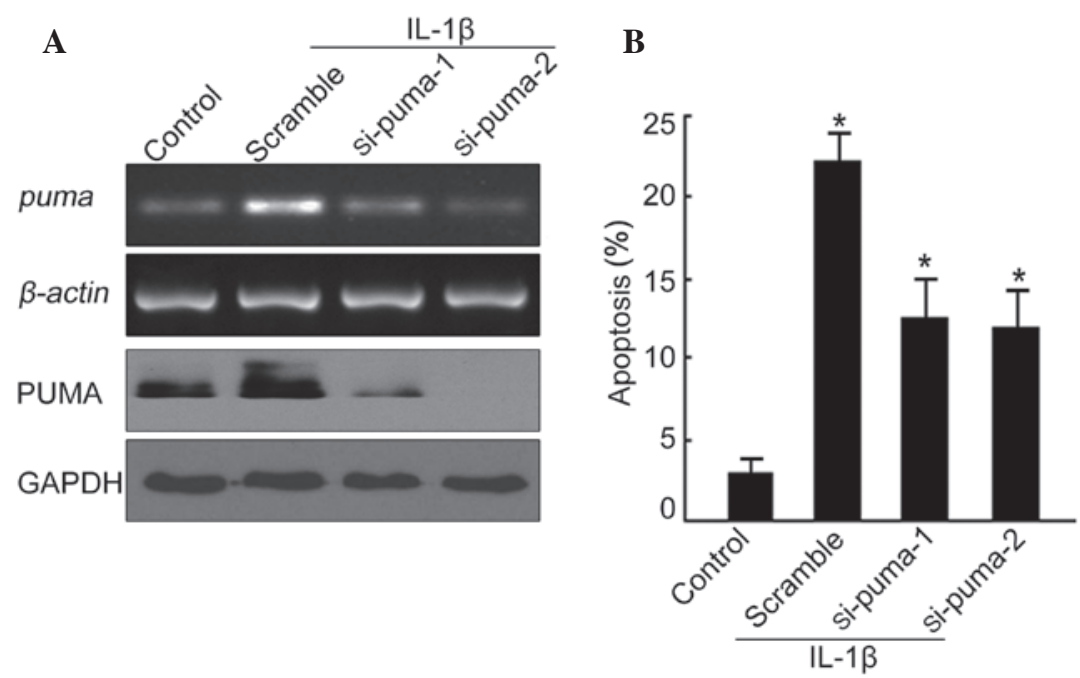

Figure 2. Upregulation of PUMA is critical for chondrocyte apoptosis. (A) Cultured mouse chondrocytes were transfected with two different PUMA siRNA fragments (si-PUMA-1 or si-PUMA-2) or scrambled siRNA for $48 \mathrm{~h}$. Then, the cells were treated with IL-1 $\beta$ (10 ng/ml) for $48 \mathrm{~h}$, collected and subjected to RT-PCR and western blotting. (B) Cells treated as in (A) were stained with Hoechst 33258 to visualize condensed nucleus for apoptosis measurements. Measurements were performed as described in Fig. 1. Quantified statistical data are presented as the mean \pm SE of at least three separate experiments. ${ }^{*}<0.05$. IL-1 $\beta$, interleukin-1 $\beta$; RT-PCR, reverse transcription-PCR.

course of IL-1 $\beta$ treatment. Together, these data indicate that IL-1 $\beta$ treatment not only has the ability to induce chondrocyte apoptosis, but also to alter PUMA protein and mRNA levels during the apoptotic process.

PUMA upregulation is critical for IL-1 $\beta$ induced chondrocyte apoptosis. Since PUMA mRNA and protein levels were upregulated in cultured chondrocytes following IL-1 $\beta$ stimulation, we then investigated whether PUMA upregulation was essential for chondrocyte apoptosis. Two different siRNA fragments specifically targeting PUMA were designed. The knockdown efficiency of each siRNA was determined by RT-PCR and western blot analysis following $72 \mathrm{~h}$ of transfection. Of the two siRNAs tested, si-PUMA-2 gave an enhanced knockdown efficiency at a concentration of $100 \mathrm{nM}$. As demonstrated in Fig. 2A (upper panel), the mRNA of PUMA was decreased by si-PUMA-1 and si-PUMA-2, respectively, as compared with the scrambled siRNA. PUMA protein levels were also significantly reduced upon treatment with si-PUMA-1 and si-PUMA-2 (Fig. 2A, lower panel), indicating a successful knockdown effect with these siRNA fragments. In addition to this, knockdown of PUMA triggered a marked decreased in the apoptotic rate in the presence of IL-1 $\beta$ (Fig. 2B). These data suggest that PUMA is critical for IL-1 $\beta$ induced chondrocyte apoptosis.

JNK/c-Jun pathway mediates upregulation of PUMA in IL-1 $\beta$ treated chondrocytes. Since the JNK/c-Jun pathway has been reported to regulate PUMA induction, it was considered that this pathway may also be involved in the induction of PUMA in an IL-1 $\beta$-induced model of apoptosis in mouse chondrocytes. Two potent and specific inhibitors of the JNK/c-Jun pathway were used; SP600125 to inhibit JNK and CEP11004 to inhibit MLK, an upstream kinase of JNK. The results identified an increased phosphorylation of JNK and c-Jun following IL-1 $\beta$ treatment, indicating activation of the JNK/c-Jun pathway (Fig. 3A). Upon the addition of $10 \mu \mathrm{M} \mathrm{SP} 600125$ or $2 \mu \mathrm{M}$ CEP11004, there was a decrease in the phosphorylation levels of c-Jun and JNK, as well as a decrease in the protein levels of PUMA (Fig. 3A). PUMA protein levels were decreased with the two inhibitors following IL-1 $\beta$ treatment (Fig. 3A). Simultaneously, PUMA mRNA was also suppressed upon treatment with SP600125 or CEP11004 in the presence of IL-1 $\beta$ (Fig. 3B).

Following this, we determined whether inhibition of the JNK/c-Jun pathway would impact the rate of apoptosis induced by IL-1 $\beta$. As demonstrated in Fig. 3C, apoptosis induced by IL-1 $\beta$ was significantly suppressed following the incubation of cells with SP600125 and CEP11004, respectively. This accumulative evidence suggests that the JNK/c-Jun pathway may be activated by IL-1 $\beta$ treatment and that it acts upstream of PUMA to mediate chondrocyte apoptosis.

PUMA and c-Jun are activated in chondrocyte tissues of $O A$ patients. To determine whether c-Jun and PUMA are upregulated in chondrocyte tissues isolated from OA patients, the expression level of c-Jun and PUMA was examined in human articular cartilage and in primary cultured chondrocytes from OA patients. Using immunohistochemistry, an increased staining of PUMA was observed in the articular cartilage of OA patients as compared with the normal tissues (Fig. 4A). Positive staining for PUMA was identified in 56\% of OA tissues tested (data not presented). Then, chondrocytes from the tissues of patients with or without a history of OA were isolated and cultured. Cell lysates were prepared and subject to western blot analysis. As compared with chondrocytes derived from healthy tissue (Fig. 4; NC1,2,3), the protein levels of c-Jun and PUMA in chondrocytes from OA patients were elevated (Fig. 4; OA1, 2, 3). Similarly, the mRNA levels for c-Jun and PUMA were also elevated in OA patients (Fig. 4, lower panel). These data demonstrated that PUMA and c-Jun are significantly upregulated in OA patients, suggesting they have a role in the apoptosis observed in the tissues of OA patients. 


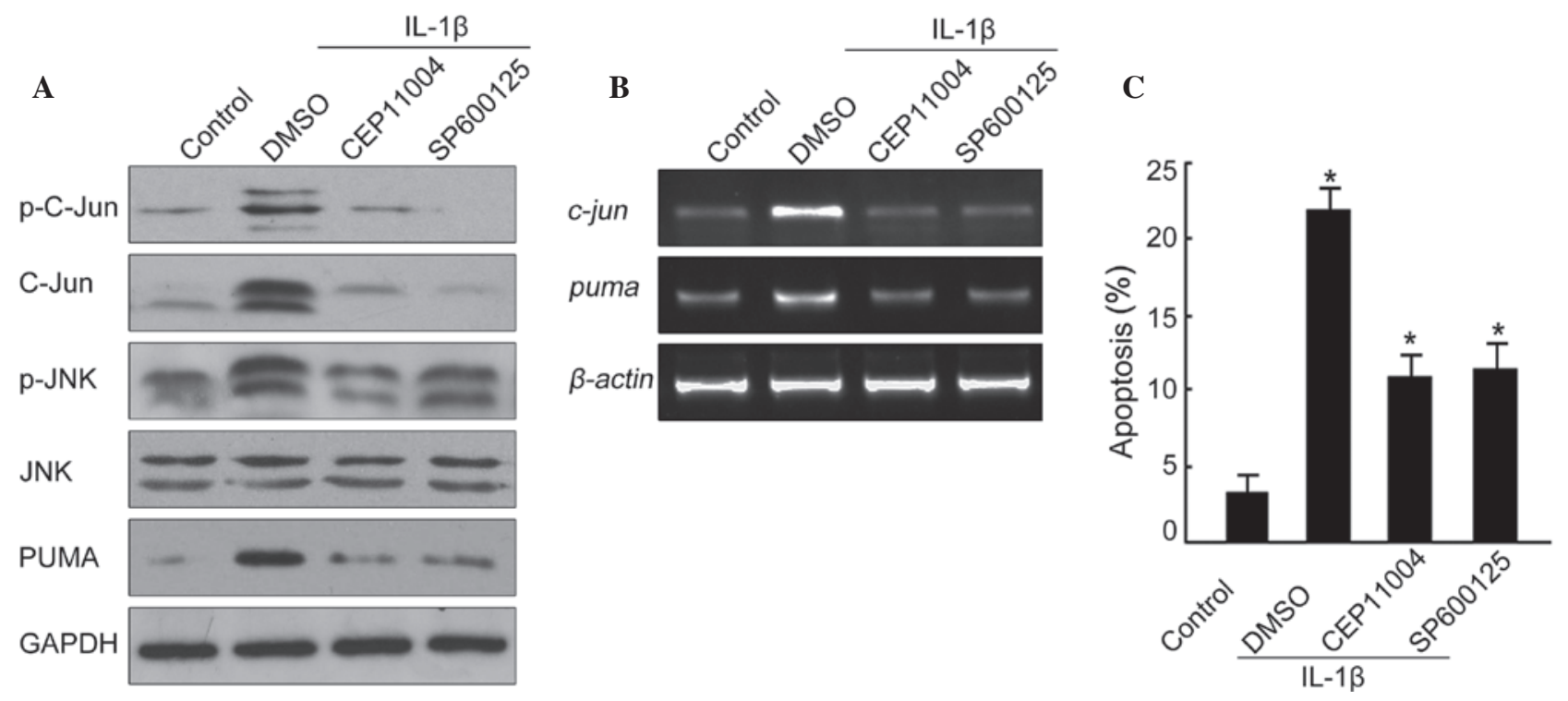

Figure 3. Inhibition of the JNK/c-Jun pathway prevents PUMA upregulation under IL-1 $\beta$ induction. Subconfluent chondrocyte monolayers were treated with IL-1 $(10 \mathrm{ng} / \mathrm{ml})$ in the presence or absence of $10 \mu \mathrm{M} \mathrm{SP} 600125$ (SP) or $2 \mu \mathrm{M} \mathrm{CEP11004} \mathrm{(CEP).} \mathrm{For} 48 \mathrm{~h}$ after treatment, samples were subjected to (A) western blotting to detect indicated proteins or (B) RT-PCR to determine mRNA level of $c$-jun and puma. The data represent the mean \pm SE of three separate experiments. (C) Cells treated as in (A) were stained with Hoechst 33258 to visualize condensed nucleus for apoptosis measurements. Measurements were performed as described in Fig. 1. Quantified statistical data are presented as the mean $\pm \mathrm{SE}$ of at least three separate experiments. ${ }^{*} \mathrm{P}<0.05$. JNK/c-Jun, c-Jun N-terminal kinase (JNK)/c-Jun; IL-1 $\beta$, interleukin-1 $\beta$; RT-PCR, reverse transcription-PCR.

A
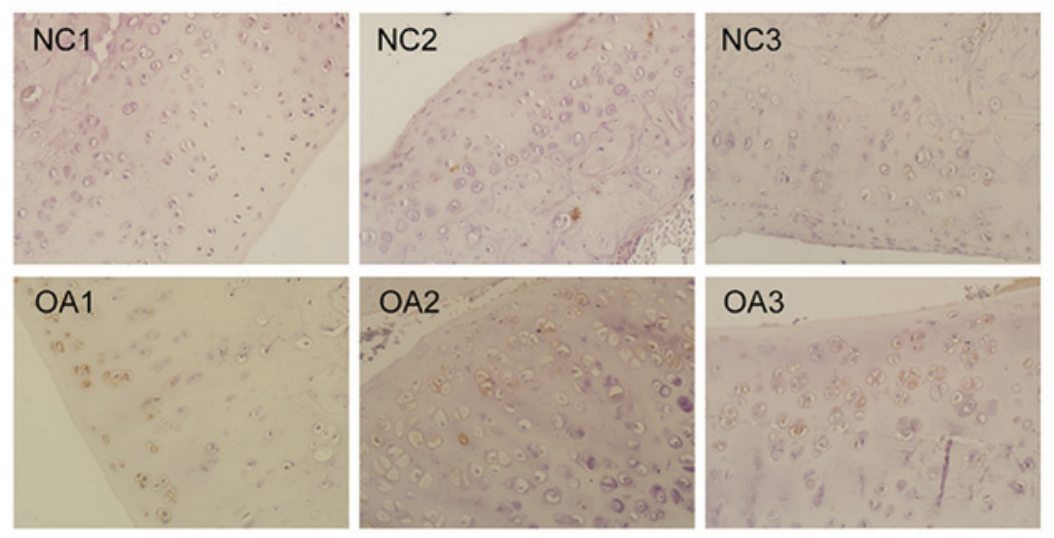

$\mathbf{B}$

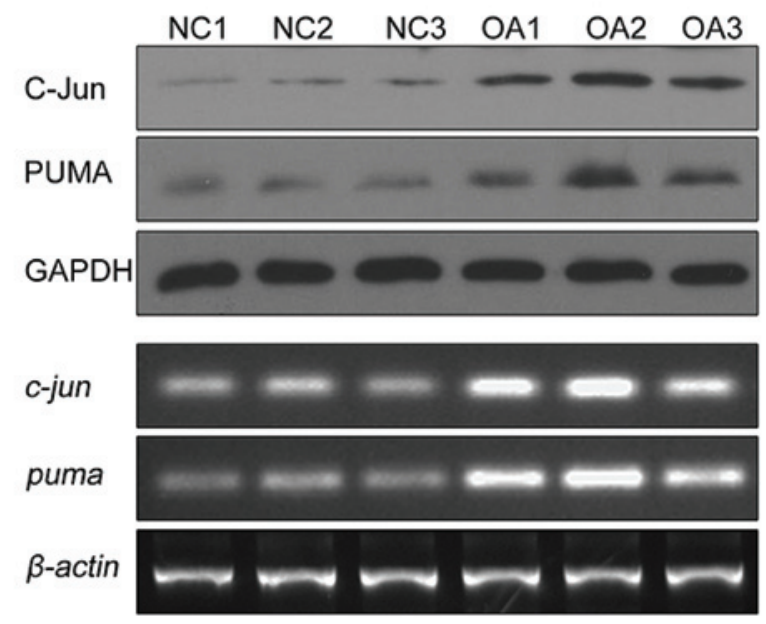

Figure 4. Upregulation of PUMA and c-Jun in chondrocyte samples from OA patients. (A) Articular cartilage samples of control patients (NC) and OA patients (OA) were isolated and embedded in paraffin. Sections from these blocks were then subjected to immunohistochemical analysis using an anti-PUMA antibody and the expression levels of PUMA were examined in the tissues. (B) Cell extracts of primary cultured human chondrocytes were immunoblotted for PUMA, c-Jun and GAPDH (loading control). Cell extracts of primary cultured human chondrocytes were subjected to RT-PCR to detect the mRNA levels of $c$-jun and puma. OA, osteoarthritis; RT-PCR, reverse transcription-PCR. 


\section{Discussion}

Numerous different types of cell death have been reported in cartilage-apoptosis, necrosis and chondroptosis. However, much controversy remains regarding which of these types predominate during OA (21). Numerous investigators have demonstrated correlations between increased rates of chondrocyte apoptosis and the severity of OA in animals (22-26) and in humans (27-32). These studies have used a wide range of analytical tools to demonstrate the correlation between apoptosis and OA, including histology, TUNEL staining, caspase-3 activation, ELISA and fluorescence-activated cell sorter analysis (FACS). Despite this, the relative importance of apoptosis in OA remains controversial because other studies have failed to confirm the presence of large numbers of apoptotic cells in OA cartilage (33). Therefore, elucidating the mechanism of cell death in cartilage may be vital for identifying new therapeutic agents for OA.

In the present study, it was demonstrated for the first time, to the best of our knowledge, that upregulated PUMA is critical for OA apoptosis in cultured chondrocytes. The results reveal that PUMA protein levels are significantly increased in human OA cartilage tissues and in chondrocytes cultured from these tissues. Additionally, chondrocytes isolated from human OA tissues also exhibit elevated PUMA mRNA levels as compared with chondrocytes derived from normal tissue. Similar to human chondrocytes, mouse chondrocytes also exhibited increased PUMA protein and mRNA levels following IL-1 $\beta$ treatment. This upregulation of PUMA implicates mitochondrial apoptosis in the progression of OA. However, we identified that Bax levels do not change in chondrocytes cultured human OA cartilage or in chondrocytes exposed to IL-1 $\beta$ (data not presented). These results may indicate the involvement of other Bcl-2 pro-apoptotic mediators, such as Bid, Bak and/or Bad. Future studies are necessary to determine the role(s) of other pro-apoptotic mediators in the pathogenesis of OA.

Activation of the JNK/c-Jun pathway has been reported in human OA cartilage and chondrocytes treated with IL-1 $\beta$ (14-16). Although PUMA is reported to be a downstream target of the JNK/c-Jun pathway in neurons, the correlation between the JNK/c-Jun pathway and PUMA in OA has not yet been determined. In the present study, it was demonstrated that c-Jun is largely upregulated in cultured human and mouse chondrocytes upon IL-1 $\beta$ treatment, which supports previous evidence in the literature. Furthermore, we identified that PUMA functions as a downstream effector of the JNK/c-Jun pathway because pharmacological inhibition of this pathway attenuated the expression of PUMA at transcriptional and post-transcriptional levels. Together, these results indicate that the JNK/c-Jun pathway may alter PUMA induction and may have a central role in the pathological process of OA.

In summary, the present study has demonstrated that PUMA is significantly upregulated in OA and the upregulation of PUMA is induced by the JNK/c-Jun pathway. This JNK/c-Jun regulation of PUMA expression is associated with cartilage damage and may provide a new therapeutic target for the development of treatment strategies for OA in the future.

\section{Acknowledgements}

The authors are grateful for financial support from the National Natural Science Foundation of China (no. 81272040 and 30600632) and the Natural Science Foundation of Guangdong Province, China (no. S2011010004808).

\section{References}

1. Goldring MB and Goldring SR: Osteoarthritis. J Cell Physiol 213: 626-634, 2007

2. Arden N and Nevitt MC: Osteoarthritis: epidemiology. Best Pract Res Clin Rheumatol 20: 3-25, 2006.

3. Hashimoto S, Ochs RL, Komiya S and Lotz M: Linkage of chondrocyte apoptosis and cartilage degradation in human osteoarthritis. Arthritis Rheum 41: 1632-1638, 1998.

4. Heraud F, Heraud A and Harmand MF: Apoptosis in normal and osteoarthritic human articular cartilage. Ann Rheum Dis 59: 959-965, 2000

5. Kobayashi M, Squires GR, Mousa A, et al: Role of interleukin-1 and tumor necrosis factor alpha in matrix degradation of human osteoarthritic cartilage. Arthritis Rheum 52: 128-135, 2005.

6. Yasuhara R, Miyamoto Y, Akaike T, et al: Interleukin-1beta induces death in chondrocyte-like ATDC5 cells through mitochondrial dysfunction and energy depletion in a reactive nitrogen and oxygen species-dependent manner. Biochem J 389: 315-323, 2005.

7. Lopez-Armada MJ, Carames B, Lires-Dean M, et al: Cytokines, tumor necrosis factor-alpha and interleukin-1beta, differentially regulate apoptosis in osteoarthritis cultured human chondrocytes. Osteoarthritis Cartilage 14: 660-669, 2006.

8. Hagemeier K, Lurbke A, Hucke S, et al: Puma, but not noxa is essential for oligodendroglial cell death. Glia 61: 1712-1723, 2013.

9. Guadagno J, Xu X, Karajgikar M, Brown A and Cregan SP: Microglia-derived TNFalpha induces apoptosis in neural precursor cells via transcriptional activation of the $\mathrm{Bcl}-2$ family member Puma. Cell Death Dis 4: e538, 2013.

10. Zheng X, He K, Zhang L and Yu J: Crizotinib induces PUMAdependent apoptosis in colon cancer cells. Mol Cancer Ther 12: 777-786, 2013.

11. Reimertz C, Kogel D, Rami A, Chittenden T and Prehn JH: Gene expression during ER stress-induced apoptosis in neurons: induction of the $\mathrm{BH} 3$-only protein Bbc3/PUMA and activation of the mitochondrial apoptosis pathway. J Cell Biol 162: 587-597, 2003.

12. Zou CG, Cao XZ, Zhao YS, et al: The molecular mechanism of endoplasmic reticulum stress-induced apoptosis in PC-12 neuronal cells: the protective effect of insulin-like growth factor I. Endocrinology 150: 277-285, 2009

13. Chang L and Karin M: Mammalian MAP kinase signalling cascades. Nature 410: 37-40, 2001.

14. Islam S, Kermode T, Sultana D, et al: Expression profile of protein tyrosine kinase genes in human osteoarthritis chondrocytes. Osteoarthritis Cartilage 9: 684-693, 2001.

15. Fan Z, Soder S, Oehler S, Fundel K and Aigner T: Activation of interleukin-1 signaling cascades in normal and osteoarthritic articular cartilage. Am J Pathol 171: 938-946, 2007.

16. Mengshol JA, Vincenti MP, Coon CI, Barchowsky A and Brinckerhoff CE: Interleukin-1 induction of collagenase 3 (matrix metalloproteinase 13) gene expression in chondrocytes requires p38, c-Jun N-terminal kinase, and nuclear factor kappaB: differential regulation of collagenase 1 and collagenase 3 . Arthritis Rheum 43: 801-811, 2000.

17. Newton R, Stevens DA, Hart LA, Lindsay M, Adcock IM and Barnes PJ: Superinduction of COX-2 mRNA by cycloheximide and interleukin-1beta involves increased transcription and correlates with increased NF-kappaB and JNK activation. FEBS Lett 418: 135-138, 1997.

18. Ray A, Shakya A and Ray BK: Inflammation-responsive transcription factors SAF-1 and c-Jun/c-Fos promote canine MMP-1 gene expression. Biochim Biophys Acta 1732: 53-61, 2005.

19. Ambacher KK, Pitzul KB, Karajgikar M, Hamilton A, Ferguson SS and Cregan SP: The JNK- and AKT/GSK3beta- signaling pathways converge to regulate Puma induction and neuronal apoptosis induced by trophic factor deprivation. PloS One 7: e46885, 2012. 
20. Zhao Z, Wang J, Tang J, et al: JNK- and Akt-mediated Puma expression in the apoptosis of cisplatin-resistant ovarian cancer cells. Biochem J 444: 291-301, 2012.

21. Zamli Z and Sharif M: Chondrocyte apoptosis: a cause or consequence of osteoarthritis? Int J Rheum Dis 14: 159-166, 2011.

22. Carames B, Taniguchi N, Otsuki S, Blanco FJ and Lotz M: Autophagy is a protective mechanism in normal cartilage, and its aging-related loss is linked with cell death and osteoarthritis. Arthritis Rheum 62: 791-801, 2010.

23. D'Lima D, Hermida J, Hashimoto S, Colwell C and Lotz M: Caspase inhibitors reduce severity of cartilage lesions in experimental osteoarthritis. Arthritis Rheum 54: 1814-1821, 2006.

24. Zemmyo M, Meharra EJ, Kuhn K, Creighton-Achermann L and Lotz M: Accelerated, aging-dependent development of osteoarthritis in alpha1 integrin-deficient mice. Arthritis Rheum 48: 2873-2880, 2003.

25. Mistry D, Oue Y, Chambers MG, Kayser MV and Mason RM: Chondrocyte death during murine osteoarthritis. Osteoarthritis Cartilage 12: 131-141, 2004.

26. Thomas CM, Fuller CJ, Whittles CE and Sharif M: Chondrocyte death by apoptosis is associated with cartilage matrix degradation. Osteoarthritis Cartilage 15: 27-34, 2007.

27. Blanco FJ, Guitian R, Vazquez-Martul E, de Toro FJ and Galdo F: Osteoarthritis chondrocytes die by apoptosis. A possible pathway for osteoarthritis pathology. Arthritis Rheum 41: 284-289, 1998.
28. Sharif M, Whitehouse A, Sharman P, Perry M and Adams M: Increased apoptosis in human osteoarthritic cartilage corresponds to reduced cell density and expression of caspase-3. Arthritis Rheum 50: 507-515, 2004.

29. Matsuo M, Nishida K, Yoshida A, Murakami T and Inoue H: Expression of caspase-3 and -9 relevant to cartilage destruction and chondrocyte apoptosis in human osteoarthritic cartilage. Acta Med Okayama 55: 333-340, 2001.

30. Kim HA, Lee YJ, Seong SC, Choe KW and Song YW: Apoptotic chondrocyte death in human osteoarthritis. J Rheumatol 27: 455-462, 2000.

31. Hashimoto S, Takahashi K, Amiel D, Coutts RD and Lotz M: Chondrocyte apoptosis and nitric oxide production during experimentally induced osteoarthritis. Arthritis Rheum 41: 1266-1274, 1998.

32. Hashimoto S, Nishiyama T, Hayashi S, et al: Role of p53 in human chondrocyte apoptosis in response to shear strain. Arthritis Rheum 60: 2340-2349, 2009.

33. Aigner T, Hemmel M, Neureiter D, et al: Apoptotic cell death is not a widespread phenomenon in normal aging and osteoarthritis human articular knee cartilage: a study of proliferation, programmed cell death (apoptosis), and viability of chondrocytes in normal and osteoarthritic human knee cartilage. Arthritis Rheum 44: 1304-1312, 2001. 